\title{
A LOCALIZATION PRINCIPLE FOR A CLASS OF ANALYTIC FUNCTIONS
}

\author{
D. A. STORVICK
}

It has been shown by Kiyoshi Noshiro [8; p. 35] that a bounded analytic function $w=f(z)$ in $|z|<1$ having radial limit values of modulus one almost everywhere satisfies a localization principle of the following type. Let $(c)$ be any circular disk: $|w-\alpha|<\rho$ lying inside $|w|<1$ whose periphery may be tangent to the circumference $|w|=1$. Denote by $\Delta$ any component of the inverse image of $(c)$ under $w=f(z)$ and by $z=z(\xi)$ a function which maps $|\xi|<1$ onto the simply connected domain $\Delta$ in a one-to-one conformal manner. Then, the function

$$
W=F(\xi)=\frac{1}{\rho}[f(z(\xi))-\alpha]
$$

is also a bounded analytic function in $|\xi|<1$ with radial limits of modulus one almost everywhere.

Maurice Heins [2; p. 455], [3] has established a localization principle for conformal mappings of type- $B l$ between Riemann surfaces.

The object of this note is to prove an extension of Noshiro's theorem.

\section{Definition 1.}

A function $w=f(z)$ which is bounded and analytic in $|z|<1$ and whose radial limit values $\lim _{r \rightarrow 1} f\left(r e^{i \theta}\right)=f^{*}\left(e^{i \theta}\right)$ exist and are of modulus one for all points $e^{i \theta}$ on $|z|=1$ except for at most a set $S$ of points $e^{i \theta}$ of linear measure zero will be called of class $(U)$ in $|z|<1$. If the possible exceptional set $S$ is of logarithmic capacity zero, cap $(S)=0, w=f(z)$ will be said to be of class $\left(U^{*}\right)$ in $|z|<1$.

For the sake of completeness we prove the following lemma.

Received June 20, 1963 and, in revised form, August 15, 1963.

Sponsored by the Mathematics Research Center, U.S. Army, Madison, Wisconsin, under Contract No.: DA-11-022-ORD-2059. 


\section{Lemma 1.}

If $w=f(z)$ is an analytic function in $|z|<1$, then every component $G(\alpha, \rho, k)$ of the open set $G(\alpha, \rho)=\{z|| z|<1| f,(z)-\alpha \mid<\rho\}$ is a simply connected domain. Proof: If $G(\alpha, \rho, k)$ is not simply connected, there exists a point $z_{0}$ in the complement of $G(\alpha, \rho, k)$ and a simple closed polygonal path $\Gamma$ contained in $G(\alpha, \rho, k)$ for which the winding number of $\Gamma$ with respect to $z_{0}$ is one, $n\left(T, z_{0}\right)$ $=+1$. Now $|f(z)-\alpha|$ has a maximum value on $I$, and $\max _{z \in \Gamma}|f(z)-\alpha|=M<\rho$ since $\Gamma \subset G(\alpha, \rho, k)$. Therefore by the maximum modulus theorem, for all $z$ in the interior of $\Gamma$, the relation $|f(z)-\alpha| \leq M<\rho$ is satisfied. This contradicts the assumption that $z_{0} \notin G(\alpha, \rho, k)$ so that $\left|f\left(z_{0}\right)-\alpha\right| \geq \rho$.

\section{Defirition 2.}

An analytic function $w=f(z)$ defined in $|z|<1$ is said to be of class $\left(U^{*}\right)$ at a point $\alpha$ if there exists a disk $|w-\alpha|<\rho$ such that for each component $G(\alpha, \rho, k)$ of $G(\alpha, \rho)=\{z|| z|<1| f,(z)-\alpha \mid<\rho\}$ the function

$$
W=F(\xi)=\frac{1}{\rho}[f(z(\xi))-\alpha]
$$

is of class $\left(U^{*}\right)$ in $|\xi|<1$ where $z=z(\xi)$ is any one-to-one conformal mapping of $|\xi|<1$ onto the simply connected domain $G(\alpha, \rho, k)$. If $w=f(z)$ is of class $\left(U^{*}\right)$ for every $\alpha$ in a domain $G$, then $w=f(z)$ is said to be locally of class $\left(U^{*}\right)$ in $G$.

The structure of domains of the type of $G(\alpha, \rho, k)$ and of their frontiers $\operatorname{Fr}(G(\alpha, \rho, k))$ for various classes of functions has been the object of extensive study by many authors. See for example the work of Lohwater [4], [5], [6], Noshiro [7], [8], Tsuji [11] and the author [10]. We now prove a lemma concerning the structure of $G(\alpha, \rho, k)$ for functions of class $\left(U^{*}\right)$. The proof is a modification of a technique used in [5] and [10].

\section{Lemma 2.}

Let $w=f(z)$ be a non-constant function of class $\left(U^{*}\right)$. Assume that for each $e^{i \beta}, \operatorname{cap}\left\{e^{i \theta} \mid f^{*}\left(e^{i \theta}\right)=e^{i \beta}\right\}=0$. Then for any $\alpha,|\alpha|<1$ and any $\rho, 0<\rho \leq$ $1-|\alpha|$ the frontier $\Gamma=\operatorname{Fr}(G(\alpha, \rho, k))$ is a Jordan curve whose intersection with $|z|=1$ is of logarithmic capacity zero.

Proof. By Lemma 1, $G(\alpha, \rho, k)$ is a simply connected domain and we now show that $\Gamma$ is locally connected at each of its points; i.e. every neighborhood $U$ of 
a point $p \in \Gamma$ contains a neighborhood $V$ of $p$ such that every point of $V \cap \Gamma$ lies in that component of $U \cap \Gamma$ which contains $p$. As a consequence, each point of $\Gamma$ is then $[12$, p. 111$]$ arcwise accessible from $G(\alpha, \rho, k)$. Now, at each point of $\Gamma$ lying interior to $|z|<1, \Gamma$ is locally connected, since it is part of a piecewise analytic arc, namely a level curve of $\log |f(z)-\alpha|$. Let $E=\Gamma \cap\{|z|$ $=1$. If $p \in E$ and if $\Gamma$ is not locally connected at $p$, then, by an elementary theorem $[12 ;$ p. 18], there exists a non-degenerate subcontinuum $N$ of $I$ containing $p$ and such that $\Gamma$ is not locally connected at any point of $N$. Since $N$ must lie on $|z|=1$, it is clear that $N$ is an arc of $|z|=1$. Furthermore, there must exist $[12 ;$ p. 18] a circular neighborhood $V$ of $p$ and a sequence of mutually disjoint components $N_{1}, N_{2}, \ldots$ of $\bar{V} \cap \Gamma$ converging to a non-degenerate limiting $\operatorname{arc} N_{n} \subset N$ containing $p$. Thus if $q$ is any interior point of $N_{0}$, every radius of $|z|<1$ drawn to $q$ must cross infinitely many of the components $N_{j}$ arbitrarily close to $q$. Along such a radius of $|z|<1$, if $f\left(r e^{i \theta}\right)$ tends to a limit of modulus one, this limit must be $\frac{\alpha}{|\alpha|}$, since $|f(z)-\alpha|=\rho \leq 1-|\alpha|$ at all points of $N_{j}$. Since this occurs at every interior point of $N_{0}$ with at most the exception of a set of logarithmic capacity zero, we violate the hypothesis that for every $e^{i \beta}$, cap $\left\{e^{i \theta} \mid f^{*}\left(e^{i \theta}\right)=e^{i \beta}\right\}=0$. Therefore $\Gamma=\operatorname{Fr}(G(\alpha \rho, k))$ must be locally connected.

We show next that the set $E=\Gamma \cap\{|z|=1\}$ is of logarithmic capacity zero. Let $M$ be the set of points on $|z|=1$ for which the radial limit values are of modulus one. We let $M=\{|z|=1\}-\tilde{M}$ and observe that cap $(\hat{M})=0$. Because of the decomposition $E=(E \cap M) \cup(E \cap \tilde{M})$ it will suffice to prove that $E \cap M$ is of logarithmic capacity zero.

We divide $M$ into two sets $M_{1}$ and $M_{2}$ in the following manner. Let $M_{1}=$ $\left\{e^{i \theta} \mid f^{*}\left(e^{i \theta}\right)=\frac{\alpha}{|\alpha|}\right\}$ and $M_{2}=M-M_{1}$. The set $M_{1}$ is by hypothesis of logarithmic capacity zero and at each point $e^{i \theta} \in M_{2} \cap E$, the radial cluster set $C_{\rho}\left(f, e^{i \theta}\right)$ is a single point $f^{*}\left(e^{i 0}\right) \neq \frac{\alpha}{|\alpha|}$. Each point $e^{i \theta} \in M_{2}$ is arcwise accessible from $G(\alpha, \rho, k)$ and the curvilinear cluster set $C_{\lambda \theta}\left(f, e^{i \theta}\right)$ along any path $\lambda \theta$ lying in $G(\alpha, \rho, k)$ and terminating at $e^{i \theta}$ is contained in $\{|w-\alpha| \leq \rho\}$. The intersection $C_{\rho}\left(f, e^{i \theta}\right) \cap C_{\lambda \theta}\left(f, e^{i \theta}\right)=\phi$ for every point $e^{i \theta} \in E \cap M_{2}$. By a result of Bagemihl [1], $E \cap M_{2}$ is at most a denumerable set and so $E \cap M$ is of logarithmic capacity zero. 
Finally, to prove that $\Gamma=\operatorname{Fr}(G(\alpha, \rho, k))$ is a Jordan curve, we must show that the complement of $\Gamma$ consists of two components $G_{1}$ and $G_{2}$ and that every point of $\Gamma$ is arcwise accessible from each of $G_{1}$ and $G_{2}$. Since we may identify $G(\alpha, \rho, k)$ with $G_{1}$, it is sufficient to show that the complement $G$ of the closure of $G(\alpha, \rho, k)$ is connected and that each point of $\Gamma$ is arcwise accessible from $G$. Now $E$ is a closed set of logarithmic capacity zero, so that between any two points of $E$ exists at least one arc of $|z|=1$ belonging to $G$. Thus if there exists a point of $G$ interior to $|z|<1$ which cannot be joined to a point of $|z|>1$ by an arc lying in $G$, there must exist a simple closed curve $r$ which lies, except for one point $q$ of $E$, entirely inside $G(\alpha, \rho, k)$ and which encloses points of $G$.

Since $f(z)$ is a bounded analytic function in the domain $\Omega$ bounded by $\gamma$ and since except for the one point $q \in \gamma, \limsup _{z \rightarrow \xi}|f(z)-\alpha|<\rho$ for all points $\xi \in \gamma=F r(\Omega)$, we see by the extended maximum principle $[8 ; \mathrm{p} .14]$ that $|f(z)-\alpha|<\rho$ for all points in $\Omega$ which contradicts the statement that there exists a point of $G$ interior to $|z|<1$ which cannot be joined to a point of $|z|>1$ by an arc lying in $G$. Hence all points of $G$ which lie in $|z|<1$ can be joined by some arc of $G$ to $|z|>1$, so that the complement of $\Gamma$ consists of two components $G_{1}$ and $G_{2}$. The accessibility of each point of $\Gamma$ from $G_{2}$ is trivial however since $E$ lies on $|z|=1$ and that part of $\Gamma$ inside $|z|<1$ consists of smooth level curves. Hence Lemma 2 is proved.

\section{THEOREM.}

Let $w=f(z)$ be a non-constant function of class $\left(U^{*}\right)$ and let $(c)$ be any circular disk $|w-a|<\rho$ lying inside $|w|<1$ whose periphery may be tangent to the circumference $|w|=1$. Denote by $G(\alpha, \rho, k)$ any component of the open set $G(\alpha, \rho)=\{z|| f(z)-a \mid<\rho\}$. Let $z=z(\xi)$ be a function which maps $|\xi|<1$ in a one-to-one conformal way onto the simply connected domain $G(\alpha, \rho, k)$. Then, if for every $e^{i \beta}$ on $|w|=1$, cap $\left\{e^{i \theta} \mid f^{*}\left(e^{i \theta}\right)=e^{i \beta}\right\}=0$, the function

$$
W=F(\xi)=\frac{1}{\rho}[f(z(\xi))-\alpha]
$$

is also of class $\left(U^{*}\right)$ and for every $e^{i \Upsilon}$ on $|W|=1, \operatorname{cap}\left\{e^{i \theta} \mid F^{*}\left(e^{i \theta}\right)=e^{i \Upsilon}\right\}=0$.

Proof. If the closure of $G(\alpha, \rho, k)$ lies in $D:|z|<1$, the theorem is clearly valid since by a well-known theorem of Carathéodory on the conformal mapping 
of Jordan domains $W=F(\xi)$ is continuous on the closed disk $|\xi| \leqq 1$.

We shall now consider the case where $G(\alpha, \rho, k)$ has at least one boundary point on $|z|=1$. We define $E(\alpha, \rho, k)=\operatorname{Fr}(G(\alpha, \rho, k) \cap\{|z|=1\})$ and observe that Lemma 2 states that cap $(E(\alpha, \rho, k))=0$.

The functions $z=z(\xi)$ and $W=F(\xi)$ are bounded analytic functions in $|\xi|<1$. Let us denote by $E_{\xi}$ the set of points $e^{i \theta}$ on $|\xi|=1$ for which the radial limit $z\left(e^{i \theta}\right)$ exists and the radial limit $\lim _{r \rightarrow 1} F\left(r e^{i \theta}\right)$ either fails to exist or if it does exist is of modulus less than one. Let $E_{z}$ denote the image of $E_{\xi}$ under $z=z(\xi)$ i.e. $E_{z}=\left\{z\left(e^{i \theta}\right) \mid e^{i \theta} \in E_{\xi}\right\}$. The set $E_{z}$ lies on $\Gamma:|z|=1$. Because $E_{z} \subset E(\alpha, \rho$, $k$ ) we can conclude that cap $E_{z}=0$. We shall now prove that the logarithmic capacity of $E_{5}$ is zero.

Let $\Gamma_{\xi}$ denote the circle $|\xi|=\frac{1}{4}$ and $\Gamma_{z}$ its image under $z=z(\xi)$. Since cap $\left(E_{z}\right)=0$, by Pfluger's theorem [9; p. 122] it follows that the extremal length of the totality of paths $\hat{J}$ joining $E_{z}$ to $I_{z}$ is infinite, $\lambda(\hat{J})=\infty$. Now as we observed above, the frontier of $G(\alpha, \rho, k)$ is locally connected and if we consider the subfamily $J_{z}$ of paths joining $E_{z}$ to $\Gamma_{z}$ and lying in $G(\alpha, \rho, k)$ then, since $J_{z} \subset \hat{J}$, it follows that $\lambda\left(J_{z}\right) \geq \lambda(\hat{J})=\infty$. Because the extremal length is a conformal invariant, we obtain $\lambda\left(J_{\xi}\right)=\infty$ where $J_{\xi}$ is the family of preimages of $J_{z}$ in $|\xi|<1$ under the transformation $z=z(\xi)$. It now follows from another application of Pfluger's theorem cited above that $\operatorname{cap}\left(E_{\xi}\right)=0$. Since $E_{\xi}$ the set of points $e^{i \theta}$ on $|\xi|=1$ such that the radial limits $\lim _{r \rightarrow 1} F\left(r e^{i \theta}\right)$ either fails to exist or is of modulus less than one, is of logarithmic capacity zero we conclude that $W=F(\xi) \in\left(U^{*}\right)$ and hence $w=f(z)$ is locally of class $\left(U^{*}\right)$ in $|z|<1$.

We now prove that for every $e^{i \Upsilon}$ on $|W|=1$, if $S_{\Upsilon}\left\{e^{i \theta} \mid F^{*}\left(e^{i \theta}\right)=e^{i \uparrow}\right\}$ then $\operatorname{cap}\left(S_{r}\right)=0$. Let $Z_{r}$ denote the image of $S_{r}$ under $z=z(\xi)$. The set $Z_{r}$ must lie on $|z|=1$ except for at most a denumerable subset in $|z|<1$. Because for every $e^{i \theta} \in Z_{\curlyvee}$ the radial limit $f^{*}\left(e^{i \theta}\right)$ exists and equals $\rho e^{i \Upsilon}+\alpha$, we observe that $\operatorname{cap}\left(Z_{\Upsilon}\right)=0$ and thus by the previous argument $\operatorname{cap}\left(S_{\Upsilon}\right)=0$ and the proof of the theorem is complete.

For conformal mappings of Riemann surfaces, in addition to proving that one may localize the notion type- $B l$, Maurice Heins also proved that maps which are locally of type- $B l$ and have as range a Riemann surface with positive ideal boundary are also globally of type- $B l$. This fact leads one to conjecture that if $w=f(z)$ is locally of class $\left(U^{*}\right)$ in $|w|<1$, then it is of class $\left(U^{*}\right)$. 
The following example, which Kiyoshi Noshiro has kindly shown to the author answers the conjecture in the negative. The example is based on a result of P. J. Myrberg (see also Noshiro [8, p. 26]). Consider a domain $\Phi$ obtained by excluding two points $\alpha_{1}, \alpha_{2}$ from the disk $|w|<1$. Let $\widetilde{\Phi}$ be the universal covering surface of $\Phi$. Let $w=f(z)$ be a function which maps the unit disk $|z|<1$ conformally onto $\hat{\Phi}$ in a one-to-one manner. Then, the perfect set $E$, on $|z|=1$, of essential singularities of $w=f(z)$ must be of linear measure zero but the capacity of $E$ must be positive. The radial cluster set $C_{\rho}\left(f, e^{i \theta}\right)$ does not lie on the circumference $|w|=1$ for every $e^{i 0} \in E$. Therefore, $E$ is considered as the exceptional set in the definition of class $(U)$. The function $w=f(z)$ is locally of class $\left(U^{*}\right)$ in $|w|<1$ because $\widetilde{\Phi}$ has only logarithmic singularities at $w=\alpha_{1}$ and $w=\alpha_{2}$.

\section{BiBLIOGRAPHY}

[1] Bagemihl, F.: Curvilinear cluster sets of arbitrary functions, Proc. Nat. Acad. Sci. (U.S.A.), 41, No. 6 (1955), pp. 379-382.

[2] Heins, M.: On the Lindelöf principle, Ann. of Math., 61 (1955), pp. 440-473.

[ 3 ] Heins, M.: Lindelöfian maps, Ann. of Math., 62 (1955), pp. 418-446.

[4] Lohwater, A. J.: The boundary values of a class of meromorphic functions, Duke Math. J., 19 (1952), pp. 243-252.

[5] Lohwater, A. J.: The reflection principle and the distribution of values of functions defined in a circle, Ann. Acad. Scient. Fennicae, AI 229 (1956), pp. 1-18.

[6] Lohwater, A. J.: The boundary behavior of meromorphic functions, Ann. Acad. Scient. Fennicae, 250/22 (1958), pp. 1-6.

[7] Noshiro, K.: Contributions to the theory of the singularities of analytic functions, Jap. J. Math., 19 (1958), pp. 299-327.

[8] Noshiro, K.: Cluster sets, Springer-Verlag, Berlin (1960).

[9] Pfluger, A.: Extremallängen und Kapazität, Comment. Math. Helvet., 29 (1955), pp. 120-131.

[10] Storvick, D. A.: On pseudo-analytic functions, Nagoya Math. J., 12 (1957), pp. 131138.

[11] Tsuji, M.: Potential theory in modern function theory, Maruzen, Tokyo (1959).

[12] Whyburn, G. T.: Analytic topology, Amer. Math. Soc. Colloq. Pub., 28 (1942).

\section{Mathematics Research Center}

U.S. Army

University of Wisconsin 